\title{
Measurements of net-charge fluctuations across various colliding systems with ALICE
}

\author{
Shaista Khan* for the ALICE Collaboration \\ Department of Physics, Aligarh Muslim University \\ Aligarh, India
}

E-mail: shaista.khan@cern.ch

Event-by-event fluctuations of conserved quantities such as electric charge, baryon number, and strangeness in ultrarelativistic heavy-ion collisions provide insight into the properties of the quarkgluon plasma and the QCD phase diagram. The net-charge fluctuations in finite phase space are usually studied using the $v_{d y n}[+,-]$ observable, which is robust against the detection efficiency losses. This observable becomes equivalent to the strongly intensive quantity $\Sigma$ with the proper scaling. In this analysis, the values of $v_{d y n}[+,-]$ are obtained with ALICE detector in various colliding systems, namely, $\mathrm{pp}$ at $\sqrt{s}=5.02 \mathrm{TeV}, \mathrm{p}-\mathrm{Pb}$ at $\sqrt{s_{\mathrm{NN}}}=5.02 \mathrm{TeV}$, and $\mathrm{Pb}-\mathrm{Pb}$ at $\sqrt{s_{\mathrm{NN}}}=2.76$ and $5.02 \mathrm{TeV}$, and $\mathrm{Xe}-\mathrm{Xe}$ at $\sqrt{s_{\mathrm{NN}}}=5.44 \mathrm{TeV}$. The observed dependence of $v_{d y n}[+,-]$ on chargedparticle density shows a regular smooth evolution of net-charge fluctuation from smaller to larger collision systems. Furthermore, the observed negative values of $v_{d y n}[+,-]$ indicate the dominance of correlation between the oppositely charged particle pairs as compared to that arising from the like-sign charge pairs. These findings are compared to predictions from HIJING, EPOS-LHC, and PYTHIA 8 models. The effect of the kinematical acceptance has also been investigated by examining the $v_{d y n}[+,-]$ dependence on transverse momentum range as well as the width of the pseudorapidity window. The effect of the hadronic resonance decays is also looked into by comparing the experimental findings with the predictions of HIJING model.

\footnotetext{
*** The European Physical Society Conference on High Energy Physics (EPS-HEP2021), *** ***26-30 July $2021 * * *$

*** Online conference, jointly organised by University Hamburg and the research center DESY ***
}

\footnotetext{
*Speaker
} 


\section{Introduction}

The interest in the studies involving event-by-event (EbyE) fluctuations in hadronic $(\mathrm{h}-\mathrm{h})$ and heavy-ion (A-A) collisions is primarily connected to the idea that the correlations and fluctuations of dynamical origin are associated with the critical phenomena of phase transitions and lead to the local and global differences between the events produced under similar initial conditions. The EbyE fluctuations of conserved quantities, such as strangeness, baryon number, and electric charge, in a limited phase space have emerged as a tool to explore the properties of the strongly interacting nuclear matter. The study of net-charge fluctuations of the produced particles on EbyE basis provides an opportunity to investigate the composition of hot and dense matter prevailing in the "fireball", created during the intermediate stage of A-A collisions which, in principle, can be characterized in the framework of QCD [1]. It has been argued that a phase transition from quark-gluon plasma (QGP) to normal hadronic matter is an entropy conserving process and therefore, fluctuations in the net-electric charge will be significantly reduced in the final state in comparison to what is expected to be observed from an hadron gas (HG) system [1-3]. Such a reduction is expected because the magnitude of net-charge fluctuations depends on the squares of the number of charges present in the system and hence strongly depends on the phase from which it originates. In a system passing through QGP phase, quarks are the charge carriers, whereas in the case of hadron gas, the charge carriers are hadrons. Thus, the charge fluctuations observed in the case of QGP with fractional charges would be smaller than those in hadron gas with integer charges [2]. Charge fluctuations are greatly influenced by volume fluctuations, that is, by the change of the system size event by event because of the impact parameter (b) variation. To overcome this effect, one has to define an observable which does not depend on volume fluctuations. The observable $v_{d y n}[+,-]$ defined in terms of first moments, variances, and a co-variance is taken as a suitable one for studying the net-charge fluctuations [4]. It is expressed as

$$
v_{d y n}[+,-]=\frac{\left\langle N_{+}\left(N_{+}-1\right)\right\rangle}{\left\langle N_{+}\right\rangle^{2}}+\frac{\left\langle N_{-}\left(N_{-}-1\right)\right\rangle}{\left\langle N_{-}\right\rangle^{2}}-2 \frac{\left\langle N_{+} N_{-}\right\rangle}{\left\langle N_{+}\right\rangle\left\langle N_{-}\right\rangle},
$$

where $N_{+}$and $N_{-}$are the event mean multiplicities of positive and negative charged particles, respectively, and angular brackets denote averaging over events. It has been pointed out [4] that $v_{d y n}[+,-]$ may be expressed in terms of integral of two-particle correlation function. Thus, $v_{d y n}[+,-]$ may be taken as a measure of relative correlation strength of,++-- , and +- particle pairs. According to Eq. 1, negative value of $v_{d y n}[+,-]$ indicates a significant contribution from correlations between particle pairs of opposite charges whereas, a positive value of $v_{d y n}[+,-]$ implies that correlations between particles of the same charge dominate the measurement. For independent emission of particles, these correlations should ideally be zero. However, in practice, a partial correlation may arise due to resonance decays, jets, and string fragmentation, etc. In this analysis, the observable $v_{d y n}[+,-]$ is considered to study the net-charge fluctuations because of its various advantages like (i) it provides the correlation strengths between positive and negative charged particles, (ii) it measures the deviation from Poissonian behaviour, and (iii) it is free from the particle detection efficiency losses. However, $v_{d y n}[+,-]$ has an intrinsic multiplicity dependence which needs to be taken into account.

As discussed in Ref. [5], $v_{d y n}[+,-]$ is independent of fluctuations of the number of sources, but it is also inversely proportional to the system size which makes it neither intensive nor extensive. 
To solve this problem, one needs to use an observable that depends neither on system volume nor on system volume fluctuations. A set of such observables has been proposed in Ref. [5] and are referred to as the strongly intensive measures. One such measure, $\Sigma$, is defined as

$$
\Sigma\left[N_{+}, N_{-}\right]=\frac{1}{\left\langle N_{+}\right\rangle+\left\langle N_{-}\right\rangle}\left[\left\langle N_{+}\right\rangle \omega_{-}+\left\langle N_{-}\right\rangle \omega_{+}-2 \operatorname{Cov}\left(N_{+}, N_{-}\right)\right],
$$

where $\omega_{+}\left(\omega_{-}\right)$is the scaled variance defined as

$$
\omega_{+}=\frac{\left\langle N_{+}^{2}\right\rangle-\left\langle N_{-}\right\rangle^{2}}{\left\langle N_{+}\right\rangle} .
$$

The strongly intensive quantity $\Sigma$ is normalized that it becomes dimensionless being unity $\left(\Sigma\left[N_{+}, N_{-}\right]=\right.$ $\left.\Sigma\left[N_{-}, N_{+}\right]=1\right)$ for independent particle emission [5]. At LHC energies, where $\left\langle N_{+}\right\rangle \approx\left\langle N_{-}\right\rangle, \Sigma$ measure and $v_{d y n}[+,-]$ can be connected using the relation

$$
\Sigma\left[N_{+}, N_{-}\right]-1=\frac{v_{d y n}[+,-]}{\frac{1}{\left\langle N_{+}\right\rangle}+\frac{1}{\left\langle N_{-}\right\rangle}} .
$$

The scaled version of $v_{d y n}[+,-]$ given by Eq. 4 is used in this analysis. It may be noted that, unlike $v_{d y n}[+,-]$, its scaled version requires efficiency correction for $\left\langle N_{+}\right\rangle$and $\left\langle N_{-}\right\rangle$. Furthermore, the scaled $v_{d y n}[+,-]$ can also be expressed in terms of second-order cumulants of net-charges $\left(\kappa_{2}\right)$, defined as

$$
\frac{\kappa_{2}\left[N_{+}-N_{-}\right]}{\left\langle N_{+}+N_{-}\right\rangle}=1+\left(\frac{v_{d y n}[+,-]}{\frac{1}{\left\langle N_{+}\right\rangle}+\frac{1}{\left\langle N_{-}\right\rangle}}\right)=\Sigma\left[N_{+}, N-\right] .
$$

Recently, a deviation from unity has been observed in the second-order cumulants of net-protons to the poissonian baseline ratio as a function of the pseudorapidity acceptance $\Delta \eta$ in the $0-5 \%$ most central $\mathrm{Pb}-\mathrm{Pb}$ collisions at $\sqrt{s_{\mathrm{NN}}}=2.76 \mathrm{TeV}$ recorded by ALICE [6]. The reason for this deviation can be accounted for the global and local baryon number conservation. These results [6] are also compared with HIJING simulations [7]. It is observed that HIJING does not describe the trend seen in data because the dominant process of short-range correlations between protons and antiprotons in HIJING is string decays.

\section{Analysis Details}

In these proceedings, the measurements of net-charge fluctuation observable, $v_{d y n}[+,-]$, were performed in $\mathrm{Pb}-\mathrm{Pb}$ collisions at $\sqrt{s_{\mathrm{NN}}}=2.76$ and $5.02 \mathrm{TeV}, \mathrm{Xe}-\mathrm{Xe}$ collisions at $\sqrt{s_{\mathrm{NN}}}=5.44 \mathrm{TeV}$, $\mathrm{p}-\mathrm{Pb}$ collisions at $\sqrt{s_{\mathrm{NN}}}=5.02 \mathrm{TeV}$, and proton-proton (pp) at $\sqrt{s}=5.02 \mathrm{TeV}$ recorded by ALICE detector at the LHC [8]. The sub-detectors used in this analysis were the Time Projection Chamber (TPC) for charged particle tracking, the Inner Tracking System (ITS) for tracking, vertexing, and triggering, and the V0 detector, covering the pseudorapidity range $-3.7<\eta<-1.7$ (V0C) and 2.8 $<\eta<5.1$ (V0A), for the event centrality estimation. Charged-particle tracks were reconstructed in the TPC within the transverse momentum range $0.2<p_{\mathrm{T}}<5.0 \mathrm{GeV} / c$ and $|\eta|<0.8$. This selection minimizes the contribution from jets (the spray of hadrons resulting from the fragmentation of a parton) and ensures a high reconstruction efficiency. 


\section{Results}

The left panel of Fig. 1 presents measurements of $v_{d y n}[+,-]$ based on Eq. 1 in various collision systems as a function of mean charged particle density, $\left\langle d N_{\mathrm{ch}} / d \eta\right\rangle$. The values of $\left\langle d N_{\mathrm{ch}} / d \eta\right\rangle$ corresponding to various centrality classes are taken from Refs. [9-12]. It may be noticed from the figure that for all the collision systems, the values of $v_{d y n}[+,-]$ are observed to be negative which indicates the dominance of the correlation between pairs of particles with opposite charge. It may also be noted that the values of $v_{d y n}[+,-]$ exhibit smooth evolution with multiplicity across various collision systems, namely, from smaller ( $\mathrm{pp}$ and $\mathrm{p}-\mathrm{Pb})$ to larger $(\mathrm{Xe}-\mathrm{Xe}$ and $\mathrm{Pb}-\mathrm{Pb})$. These results are compared with predictions from HIJING [7], EPOS-LHC [13], and PYTHIA8 Monash tune [14] Monte Carlo (MC) models. The trend of variations of $v_{d y n}[+,-]$ with $\left\langle d N_{\mathrm{ch}} / d \eta\right\rangle$ is nicely reproduced by the considered MC models. However, further conclusions cannot be drawn due to the intrinsic multiplicity dependence of $v_{d y n}[+,-]$.
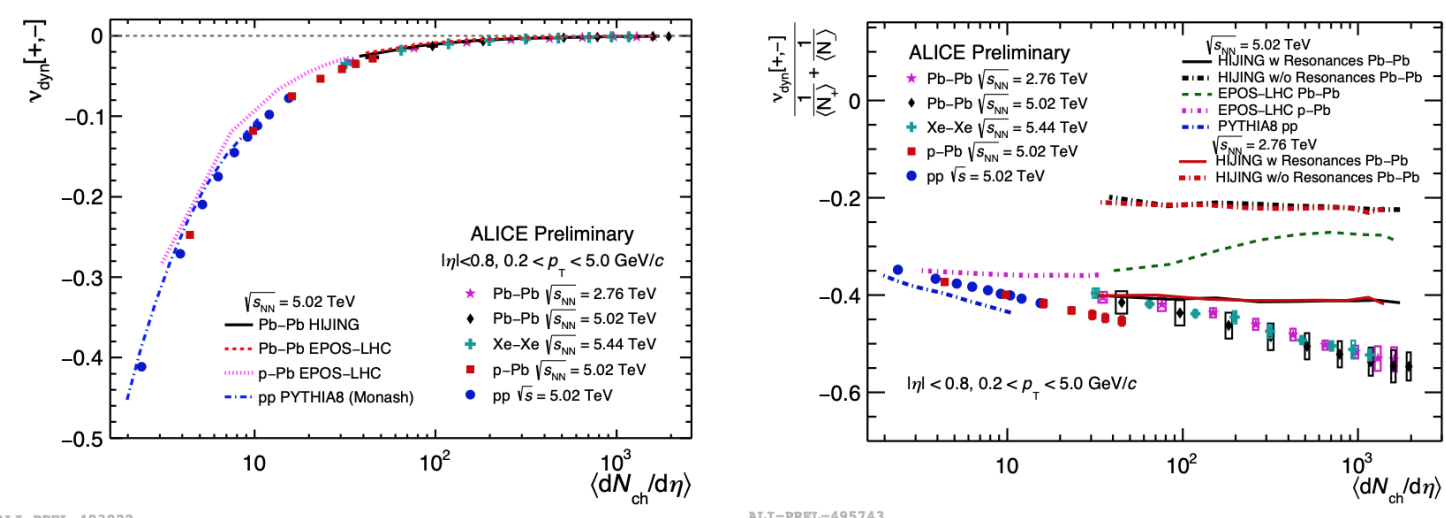

Figure 1: Left: Results for net-charge fluctuations as a function of charged particle density, $\left\langle d N_{\mathrm{ch}} / d \eta\right\rangle$, Right: Multiplicity dependence of $v_{d y n}[+,-]$ scaled by $1 /\left(\frac{1}{\left\langle N_{+}\right\rangle}+\frac{1}{\left\langle N_{-}\right\rangle}\right)$.

The right panel of Fig. 1 shows $v_{d y n}[+,-]$ scaled by $1 /\left(\frac{1}{\left\langle N_{+}\right\rangle}+\frac{1}{\left\langle N_{-}\right\rangle}\right)$as a function of $\left\langle d N_{\mathrm{ch}} / d \eta\right\rangle$. The observed scaled $v_{d y n}[+,-]$ values show a multiplicity dependence. This multiplicity dependence may arise due to the charge conservation effect and resonance contributions coupled with radial flow. It is also observed from the figure that HIJING predicts no multiplicity dependence of $v_{d y n}[+,-]$, which is expected because the model treats nucleus-nucleus collisions as the superpositions of independent nucleon-nucleon interactions and does not incorporate collective effects. The model, however, predicts that resonance decays contribute significantly to the net-charge fluctuations. This is clearly reflected from Fig. 1 (right), where the scaled values of $v_{d y n}[+,-]$ from HIJING are presented for resonance decays switched off and on. The EPOS-LHC, which is based on core-corona model, fails to describe the trend of variation of $v_{d y n}[+,-]$ with $\left\langle d N_{\mathrm{ch}} / d \eta\right\rangle$ shown by the data. Furthermore, for pp collisions, PYTHIA8 Monash qualitatively describes the measurements.

In order to examine the kinematical acceptance effect, the scaled values of $v_{d y n}[+,-]$ are presented for $\eta$ windows of different widths. The windows are chosen to be symmetric around $\eta=0$, and their width is increased in steps of 0.2 , starting from 0.2 , until the considered $\eta$ range is covered. Variations of $v_{d y n}[+,-]$ as a function of $\Delta \eta$ for the $0-5 \%$ and $70-80 \%$ centrality classes are shown 
in left panel of Fig. 2. The HIJING values are also shown in the figure. The values of $v_{d y n}[+,-]$ are observed to decrease with increasing $\Delta \eta$. For small $\Delta \eta$ windows, the observable approaches Poissonian limit.
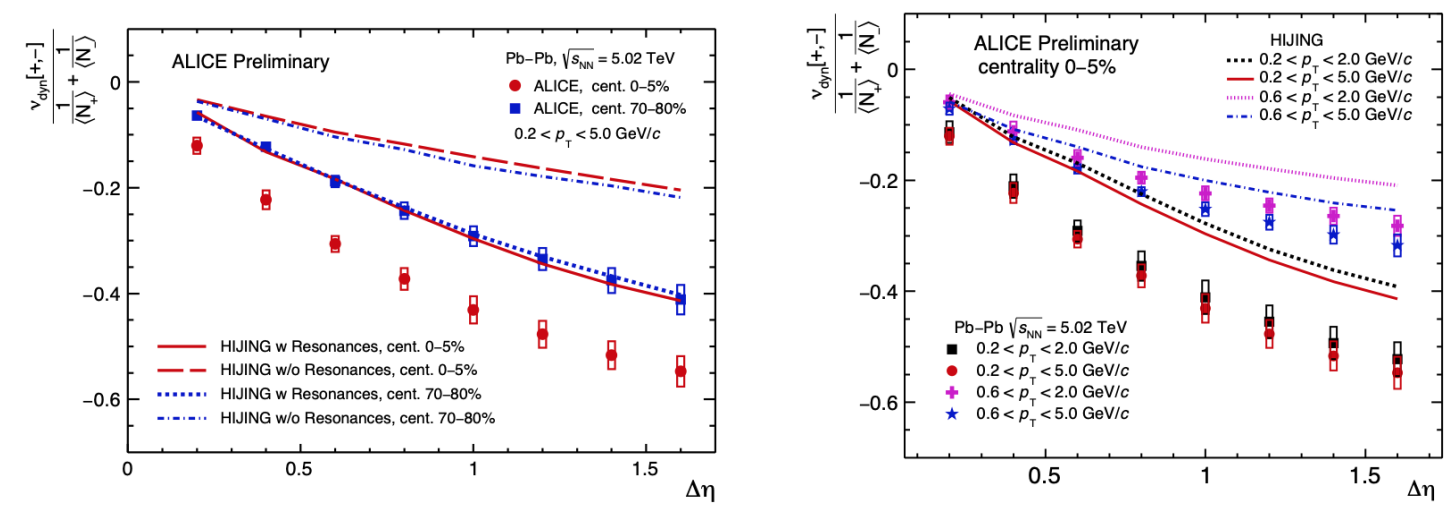

ALI-PREL-495747

Figure 2: Left: Pseudorapidity dependence of scaled $v_{d y n}[+,-]$ in $\mathrm{Pb}-\mathrm{Pb}$ collisions at $\sqrt{s_{\mathrm{NN}}}=5.02 \mathrm{TeV}$ for two centrality classes; Right: $p_{\mathrm{T}}$ dependence of scaled $v_{d y n}[+,-]$ in $\mathrm{Pb}-\mathrm{Pb}$ collisions at $\sqrt{s_{\mathrm{NN}}}=5.02 \mathrm{TeV}$ for most central events.

The influence of resonance decays and minijets on $v_{d y n}[+,-]$ has also been studied by carrying out the analysis in different $p_{\mathrm{T}}$ intervals. The values of $v_{d y n}[+,-]$ are calculated for the $p_{\mathrm{T}}$ ranges, (i) $0.2<p_{\mathrm{T}}<2.0 \mathrm{GeV} / c$, (ii) $0.2<p_{\mathrm{T}}<5.0 \mathrm{GeV} / c$, (iii) $0.6<p_{\mathrm{T}}<2.0 \mathrm{GeV} / c$, and (iv) $0.6<p_{\mathrm{T}}<$ $5.0 \mathrm{GeV} / c$. The results together with HIJING predictions in the same $p_{\mathrm{T}}$ intervals are presented in the right panel of Fig. 2. The $p_{\mathrm{T}}$ dependence of scaled $v_{d y n}[+,-]$ suggests additional contributions from resonances and minijets to this observable for $p_{\mathrm{T}}>0.6 \mathrm{GeV} / c$. The HIJING model shows qualitatively similar behaviour as exhibited by the data.

A comparison of ALICE results of $v_{d y n}[+,-]$ scaled by $\left\langle d N_{\mathrm{ch}} / d \eta\right\rangle$ for the considered collision systems with the STAR results [15] is displayed in Fig. 3. The measured $v_{d y n}[+,-] \times\left\langle d N_{\mathrm{ch}} / d \eta\right\rangle$ values for various data sets show qualitatively similar trends of variations with $\left\langle d N_{\mathrm{ch}} / d \eta\right\rangle$ as observed by STAR collaboration for Au-Au collisions at $\sqrt{s_{\mathrm{NN}}}=19.6,62.4,130$, and $200 \mathrm{GeV}$. However, the quantitative difference between the ALICE and STAR results might be due to the different $\eta$ coverage and the criteria of primary particle selection adopted in the two experiments.

\section{Conclusions}

Dynamical net-charge fluctuations in $\mathrm{pp}, \mathrm{p}-\mathrm{Pb}, \mathrm{Pb}-\mathrm{Pb}$, and $\mathrm{Xe}-\mathrm{Xe}$ collisions at $\mathrm{LHC}$ energies are studied in terms of the observable $v_{d y n}[+,-]$. A smooth evolution of such fluctuations with multiplicity are observed from smaller to larger collision systems. The $v_{d y n}[+,-]$ values, if scaled with mean multiplicities, show increasing correlations with increasing multiplicity from smaller to larger collision systems. This indicates that the charge conservation effect and resonance contribution coupled with radial flow might be responsible for such behaviour of the data. The MC models fail to describe the ALICE data quantitatively. For instance, HIJING shows no multiplicity dependence, while EPOS-LHC exhibits an opposite trend as the one observed in data. However, 


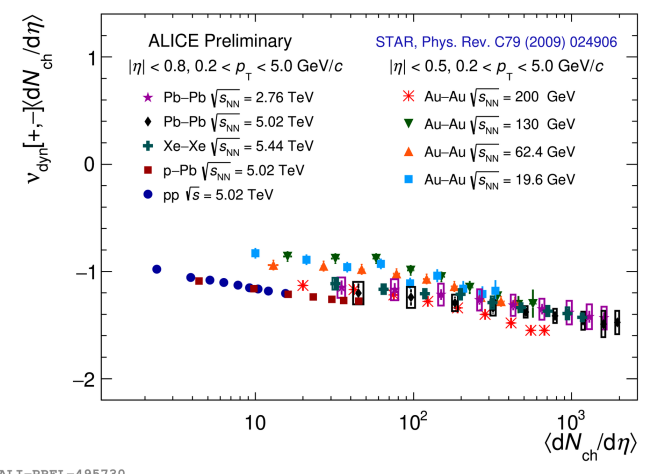

Figure 3: Comparison of $v_{d y n}[+,-]$ scaled by $\left\langle d N_{\mathrm{ch}} / d \eta\right\rangle$ with measurements at lower energies.

PYTHIA8 Monash qualitatively describes the trend of pp data. The results also reveal that significant contributions to the fluctuation observable, $v_{d y n}[+,-]$, come from the decay of hadronic resonances.

\section{References}

[1] S. Ghosh et al., Net-charge fluctuation in Au+Au collisions at energies available at the Facility for Antiproton and Ion Research using the UrQMD model, Phys. Rev. C 96 (2017) 024912 [nucl-th/1805.00790].

[2] S. Jeon and V. Koch, Charged Particle Ratio Fluctuation as a Signal for Quark-Gluon Plasma, Phys. Rev. Lett. 85 (2000) 2076 [hep-ph/0003168].

[3] M. Asakawa et al., Fluctuation Probes of Quark Deconfinement, Phys. Rev. Lett. 85 (2000) 2072 [hep-ph/0003169].

[4] C. Pruneau et al., Methods for the study of particle production fluctuations, Phys. Rev. C 66 (2002) 044904 [nucl-ex/0204011].

[5] M. I. Gorenstein and M. Gazdzicki, Strongly Intensive Quantities, Phys. Rev. C 84 (2011) 014904 [nucl-th/1101.4865].

[6] ALICE Collaboration, S. Acharya et al., Global baryon number conservation encoded in net-proton fluctuations measured in Pb-Pb collisions at $\sqrt{s_{N N}}=2.76 \mathrm{TeV}$, Phys. Lett. B 807 (2020) 135564 [nucl-ex/1910.14396].

[7] M. Gyulassy and X. N. Wang, HIJING 1.0: A Monte Carlo program for parton and particle production in high-energy hadronic and nuclear collisions, Comput.Phys. Commun. 83 (1994) 307 [nucl-th/9502021].

[8] ALICE Collaboration, K. Aamodt et al., The ALICE experiment at the CERN LHC, JINST 3 (2008) S08002 .

[9] ALICE Collaboration, K. Aamodt et al., Centrality Dependence of the Charged-Particle Multiplicity Density at Midrapidity in Pb-Pb Collisions at $\sqrt{s_{N N}}=2.76 \mathrm{TeV}$, Phys. Rev. Lett. 106 (2011) 032301 [nucl-ex/1012.1657]. 
[10] ALICE Collaboration, J. Adam et al., Centrality dependence of the charged-particle multiplicity density at mid-rapidity in Pb-Pb collisions at $\sqrt{s_{N N}}=5.02 \mathrm{TeV}$, Phys. Rev. Lett. 116 (2016) 222302 [nucl-ex/1512.06104].

[11] ALICE Collaboration, S. Acharya et al., Centrality and pseudorapidity dependence of the charged-particle multiplicity density in Xe-Xe collisions at $\sqrt{s_{N N}}=5.44 \mathrm{TeV}$, Phys. Lett. B 790 (2019) 35 [nucl-ex/1805 . 04432].

[12] ALICE Collaboration, B. Abelev et al., Multiplicity Dependence of Pion, Kaon, Proton and Lambda Production in p-Pb Collisions at $\sqrt{s_{N N}}=5.02 \mathrm{TeV}$, Phys. Lett. B 728 (2014) 25 [nucl-ex/1307.6796].

[13] T. Pierog et al., EPOS LHC: Test of collective hadronization with data measured at the CERN Large Hadron Collider, Phys. Rev. C 92 (2015) 034906 [hep-ph/306.0121].

[14] P. Skands et al., Tuning PYTHIA 8.1: the Monash 2013 tune, Eur. Phys. J. C 74 (2014) 3024 [hep-ph/1404.5630].

[15] STAR Collaboration, B. I. Abelev et al., Beam-Energy and System-Size Dependence of Dynamical Net Charge Fluctuations, Phys. Rev. C 79 (2009) 024906 [nucl-ex/0807. 3269]. 